\title{
Weighted DFT Based Blur Invariants for Pattern Recognition
}

\author{
Ville Ojansivu and Janne Heikkilä \\ Machine Vision Group, Department of Electrical and Information Engineering, \\ University of Oulu, PO Box 4500, 90014, Finland \\ $\{$ vpo,jth\}@ee.oulu.fi
}

\begin{abstract}
Recognition of patterns in blurred images can be achieved without deblurring of the images by using image features that are invariant to blur. All known blur invariants are based either on image moments or Fourier phase. In this paper, we introduce a method that improves the results obtained by existing state of the art blur invariant Fourier domain features. In this method, the invariants are weighted according to their reliability, which is proportional to their estimated signal-tonoise ratio. Because the invariants are non-linear functions of the image data, we apply a linearization scheme to estimate their noise covariance matrix, which is used for computation of the weighted distance between the images in classification. We applied similar weighting scheme to blur and blur-translation invariant features in the Fourier domain. For illustration we did experiments also with other Fourier and spatial domain features with and without weighting. In the experiments, the classification accuracy of the Fourier domain invariants was increased by up to $20 \%$ through the use of weighting.
\end{abstract}

\section{Introduction}

Recognition of objects and patterns in images is a fundamental part of computer vision with numerous applications. The task is difficult as the objects rarely look similar in different conditions. Images may contain various artefacts such as geometrical and convolutional degradations. In an ideal situation, an image analysis system should be invariant to the degradations.

We are specifically interested in invariance to image blurring, which is one type of image degradation. Typically, blur is caused by motion between the camera and the scene, an out of focus of the lens, or atmospheric turbulence. Although most of the research on invariants has been devoted to geometrical invariance [1, there are also papers considering blur invariance [2 3456]. An alternative approach to blur insensitive recognition would be deblurring of the images, followed by recognition of the sharp pattern. However, deblurring is an ill-posed problem which often results in new artefacts in images [7].

All of the blur invariant features introduced thus far are invariant to uniform centrally symmetric blur. In an ideal case, the point spread functions (PSF) of linear motion, out of focus, and atmospheric turbulence blur for a long exposure 
are centrally symmetric [7]. The invariants are computed either in the spatial domain 234] or in the Fourier domain [56], and have also geometrical invariance properties.

For blur and blur-translation invariants, the best classification results are obtained using the invariants proposed in [5, which are computed from the phase spectrum or bispectrum phase of the images. The former are called phase blur invariants (PBI) and the latter, which are also translation invariant, are referred to as phase blur-translation invariants (PBTI). These methods are less sensitive to noise compared to image moment based blur-translation invariants 2] and are also faster to compute using FFT. Also other Fourier domain blur invariants have been proposed, which are based on a tangent of the Fourier phase 2 and are referred as the phase-tangent invariants in this paper. However, these invariants tend to be very unstable due to the properties of the tangent-function. PBTIs are also the only combined blur-translation invariants in the Fourier domain. Because all the Fourier domain invariants utilize only the phase, they are additionally invariant to uniform illumination changes.

The stability of the phase-tangent invariants was greatly improved in [8] by using a statistical weighting of the invariants based on the estimated effect of image noise. Weighting improved also the results of moment invariants slightly. In this paper, we utilize a similar weighting scheme for the PBI and PBTI features. We also present comparative experiments between all the blur and blur-translation invariants, with and without weighting.

\section{Blur Invariant Features Based on DFT Phase}

The blur invariant features introduced in [5] assume that the blurred images $g(\mathbf{n})$ are generated by a linear shift invariant (LSI) process which is given by the convolution of the ideal image $f(\mathbf{n})$ with a point spread function (PSF) of the blur $h(\mathbf{n})$, namely

$$
g(\mathbf{n})=(f * h)(\mathbf{n}),
$$

where $\mathbf{n}=\left[n_{1}, n_{2}\right]^{T}$ denotes discrete spatial coordinates. It is further assumed that $h(\mathbf{n})$ is centrally symmetric, that is $h(\mathbf{n})=h(-\mathbf{n})$. In practice, images contain also noise, whereupon the observed image becomes

$$
\hat{g}(\mathbf{n})=g(\mathbf{n})+w(\mathbf{n}),
$$

where $w(\mathbf{n})$ denotes additive noise.

In the Fourier domain, the same blurring process is given by a multiplication. By neglecting the noise term, this is expressed by

$$
G(\mathbf{u})=F(\mathbf{u}) \cdot H(\mathbf{u}),
$$

where $G(\mathbf{u}), F(\mathbf{u})$, and $H(\mathbf{u})$ are the 2-D discrete Fourier transforms (DFT) of the observed image, the ideal image, and the PSF of the blur, and where 
$\mathbf{u}=\left[u_{1}, u_{2}\right]^{T}$ is a vector of frequencies. The DFT phase $\phi_{g}(\mathbf{u})$ of the observed image is given by the sum of the phases of the ideal image and the PSF, namely

$$
\phi_{g}(\mathbf{u})=\phi_{f}(\mathbf{u})+\phi_{h}(\mathbf{u}) .
$$

Because $h(\mathbf{n})=h(-\mathbf{n})$, the $H(\mathbf{u})$ is real valued and $\phi_{h}(\mathbf{u}) \in\{0, \pi\}$. Thus, $\phi_{g}(\mathbf{u})$ may deviate from $\phi_{f}(\mathbf{u})$ by angle $\pi$. This effect of $\phi_{h}(\mathbf{u})$ can be cancelled by doubling the phase modulo $2 \pi$, resulting to the phase blur invariants (PBI)

$$
\begin{aligned}
\mathcal{B}\left(\mathbf{u}_{i}\right) \equiv \mathcal{B}\left(\mathbf{u}_{i}, G\right) & =2 \phi_{g}\left(\mathbf{u}_{i}\right) \bmod 2 \pi \\
& =2 \arctan \left(\frac{p_{i}^{0}}{p_{i}^{1}}\right) \bmod 2 \pi,
\end{aligned}
$$

where $\mathbf{p}_{i}=\left[p_{i}^{0}, p_{i}^{1}\right]=\left[\operatorname{Im}\left\{G\left(\mathbf{u}_{i}\right)\right\}, \operatorname{Re}\left\{G\left(\mathbf{u}_{i}\right)\right\}\right]$, and where $\operatorname{Im}\{\cdot\}$ and $\operatorname{Re}\{\cdot\}$ denote the real and imaginary parts of a complex number.

In [5], a shift invariant bispectrum slice of the observed image, defined by

$$
\Psi(\mathbf{u})=G(\mathbf{u})^{2} G^{*}(2 \mathbf{u}),
$$

was used to obtain blur and translation invariants. The phase of the bispectrum slice is expressed by

$$
\phi_{\Psi}(\mathbf{u})=2 \phi_{g}(\mathbf{u})-\phi_{g}(2 \mathbf{u}) .
$$

Also the phase of the bispectrum slice is made invariant to blur by doubling it modulo $2 \pi$. This results in combined phase blur-translation invariants (PBTI), given by

$$
\begin{aligned}
\mathcal{T}\left(\mathbf{u}_{i}\right) \equiv \mathcal{T}\left(\mathbf{u}_{i}, G\right) & =2\left[2 \phi_{g}\left(\mathbf{u}_{i}\right)-\phi_{g}\left(2 \mathbf{u}_{i}\right)\right] \bmod 2 \pi \\
& =2\left[2 \arctan \left(\frac{p_{i}^{0}}{p_{i}^{1}}\right)-\arctan \left(\frac{q_{i}^{0}}{q_{i}^{1}}\right)\right] \bmod 2 \pi,
\end{aligned}
$$

where $\mathbf{p}_{i}$ is as above while $\mathbf{q}_{i}=\left[q_{i}^{0}, q_{i}^{1}\right]=\left[\operatorname{Im}\left\{G\left(2 \mathbf{u}_{i}\right)\right\}, \operatorname{Re}\left\{G\left(2 \mathbf{u}_{i}\right)\right\}\right]$.

\section{$3 \quad$ Weighting of the Blur Invariant Features}

For image recognition purposes, the similarity between two blurred and noisy images $\hat{g}_{1}(\mathbf{n})$ and $\hat{g}_{2}(\mathbf{n})$ can be deduced based on some distance measure between the vectors of PBI or PBTI features computed for the images. Because the values of the invariants are affected by the image noise, the image classification result can be improved if the contribution of the individual invariants to the distance measure is weighted according to their noisiness. In this section, we introduce a method for computation of a weighted distance between the PBI or PBTI feature vectors based on the estimated signal-to-noise ratio of the features. The method is similar to the one given in paper 8 for the moment invariants and phase-tangent invariants. The weighting is done by computing a Mahalanobis distance between the 
feature vectors of distorted images $\hat{g}_{1}(\mathbf{n})$ and $\hat{g}_{2}(\mathbf{n})$ as shown in Sect. 3.1. For the computation of the Mahalanobis distance, we need the covariance matrices of the PBI and PBTI features, which are derived in Sects. 3.2 and 3.3, respectively.

It is assumed that invariants (5) and (8) are computed for noisy $N$-by- $N$ image $\hat{g}(\mathbf{n})$ of which DFT is given by

$$
\begin{aligned}
\hat{G}(\mathbf{u}) & =\sum_{\mathbf{n}}\{g(\mathbf{n})+w(\mathbf{n})\} e^{-2 \pi j\left(\mathbf{u}^{T} \mathbf{n}\right) / N} \\
& =G(\mathbf{u})+\sum_{\mathbf{n}} w(\mathbf{n}) e^{-2 \pi j\left(\mathbf{u}^{T} \mathbf{n}\right) / N},
\end{aligned}
$$

where noise $w(\mathbf{n})$ is assumed to be zero-mean independent and identically distributed with variance $\sigma^{2}$. These noisy invariants are denoted by $\hat{\mathcal{B}}\left(\mathbf{u}_{i}\right) \equiv$ $\mathcal{B}\left(\mathbf{u}_{i}, \hat{G}\right)$ and $\hat{\mathcal{T}}\left(\mathbf{u}_{i}\right) \equiv \mathcal{T}\left(\mathbf{u}_{i}, \hat{G}\right)$. We use also the following notation: $\hat{\mathbf{p}}_{i}=$ $\left[\hat{p}_{i}^{0}, \hat{p}_{i}^{1}\right]=\left[\operatorname{Im}\left\{\hat{G}\left(\mathbf{u}_{i}\right)\right\}, \operatorname{Re}\left\{\hat{G}\left(\mathbf{u}_{i}\right)\right\}\right]$ and $\hat{\mathbf{q}}_{i}=\left[\hat{q}_{i}^{0}, \hat{q}_{i}^{1}\right]=\left[\operatorname{Im}\left\{\hat{G}\left(2 \mathbf{u}_{i}\right)\right\}, \operatorname{Re}\left\{\hat{G}\left(2 \mathbf{u}_{i}\right)\right\}\right]$. As only the relative effect of noise is considered, $\sigma^{2}$ does not have to be known.

\subsection{Weighted Distance between the Feature Vectors}

Weighting of the invariant features is done by computing a Mahalanobis distance between the feature vectors. Mahalanobis distance is then used as a similarity measure in classification of the images. Mahalanobis distance is computed by using the sum $\mathbf{C}_{S}=\mathbf{C}_{T}^{\left(\hat{g}_{1}\right)}+\mathbf{C}_{T}^{\left(\hat{g}_{2}\right)}$ of the covariance matrices of the PBI or PBTI features of images $\hat{g}_{1}(\mathbf{n})$ and $\hat{g}_{2}(\mathbf{n})$, and is given by

$$
\text { distance }=\mathbf{d}^{T} \mathbf{C}_{S}^{-1} \mathbf{d}
$$

where $\mathbf{d}=\left[d_{0}, d_{1}, \ldots, d_{N_{T}-1}\right]^{T}$, contains the unweighted differences of the invariants for images $\hat{g}_{1}(\mathbf{n})$ and $\hat{g}_{2}(\mathbf{n})$ in the range $[-\pi, \pi]$, which are expressed by

$$
d_{i}= \begin{cases}\alpha_{i}-2 \pi & \text { if } \alpha_{i}>\pi \\ \alpha_{i} & \text { otherwise, and }\end{cases}
$$

where $\alpha_{i}=\left[\hat{\mathcal{B}}\left(\mathbf{u}_{i}\right)^{\left(\hat{g}_{1}\right)}-\hat{\mathcal{B}}\left(\mathbf{u}_{i}\right)^{\left(\hat{g}_{2}\right)} \bmod 2 \pi\right]$ for PBIs and $\alpha_{i}=\left[\hat{\mathcal{T}}\left(\mathbf{u}_{i}\right)^{\left(\hat{g}_{1}\right)}-\right.$ $\left.\hat{\mathcal{T}}\left(\mathbf{u}_{i}\right)^{\left(\hat{g}_{2}\right)} \bmod 2 \pi\right]$ for PBTIs. $\hat{\mathcal{B}}(\mathbf{u})^{\left(\hat{g}_{k}\right)}$ and $\hat{\mathcal{T}}(\mathbf{u})^{\left(\hat{g}_{k}\right)}$ denote invariants (5) and (8), respectively, for image $\hat{g}_{k}(\mathbf{n})$.

Basically the modulo operator in (5) and (8) can be omitted due to the use of the same operator in computation of $\alpha_{i}$. The modulo operator of (15) and (8) can be neglected also in the computation of the covariance matrices in Sects. 3.2 and 3.3 .

\subsection{The Covariances of the PBI Features}

The covariance matrix of the PBIs (5) can not be computed directly as they are a non-linear function of the image data. Instead, we approximate the $N_{T}$-by- $N_{T}$ covariance matrix $\mathbf{C}_{T}$ of $N_{T}$ invariants $\hat{\mathcal{B}}\left(\mathbf{u}_{i}\right), i=0,1, \ldots, N_{T}-1$, using linearization 


$$
\mathbf{C}_{T} \approx \mathbf{J} \cdot \mathbf{C} \cdot \mathbf{J}^{T}
$$

where $\mathbf{C}$ is $2 N_{T}$-by- $2 N_{T}$ covariance matrix of the elements of vector $\mathbf{P}=\left[\hat{p}_{0}^{0}, \hat{p}_{0}^{1}, \hat{p}_{1}^{0}, \hat{p}_{1}^{1}, \cdots, \hat{p}_{N_{T}-1}^{0}, \hat{p}_{N_{T}-1}^{1}\right]$, and $\mathbf{J}$ is a Jacobian matrix. It can be shown, that due to the orthogonality of the Fourier transform, the covariance terms of $\mathbf{C}$ are zero and the $2 N_{T}$-by- $2 N_{T}$ covariance matrix is diagonal resulting in

$$
\mathbf{C}_{T} \approx \frac{N^{2}}{2} \sigma^{2} \mathbf{J} \cdot \mathbf{J}^{T}
$$

The Jacobian matrix is block diagonal and given by

$$
\mathbf{J}=\left[\begin{array}{cccc}
\mathbf{J}_{0} & 0 & \cdots & 0 \\
0 & \mathbf{J}_{1} & \cdots & 0 \\
\vdots & \vdots & \ddots & \vdots \\
0 & 0 & \cdots & \mathbf{J}_{N_{T}-1}
\end{array}\right]
$$

where $\mathbf{J}_{i}, i=0, \ldots, N_{T}-1$ contains the partial derivatives of the invariants $\mathcal{B}\left(\mathbf{u}_{i}\right)$ with respect to $\hat{p}_{i}^{0}$ and $\hat{p}_{i}^{1}$, namely

$$
\begin{aligned}
\mathbf{J}_{i} & =\left[\frac{\partial \hat{\mathcal{B}}\left(\mathbf{u}_{i}\right)}{\partial \hat{p}_{i}^{0}}, \frac{\partial \hat{\mathcal{B}}\left(\mathbf{u}_{i}\right)}{\partial \hat{p}_{i}^{1}}\right] \\
& =\left[\frac{2 \hat{p}_{i}^{1}}{c_{i}}, \frac{-2 \hat{p}_{i}^{0}}{c_{i}}\right],
\end{aligned}
$$

where $c_{i}=\left[\hat{p}_{i}^{0}\right]^{2}+\left[\hat{p}_{i}^{1}\right]^{2}$. Notice that the modulo operator in (5) does not have any effect on the derivatives of $\mathcal{B}(\mathbf{u})$, and it can be omitted.

\subsection{The Covariances of the PBTI Features}

For PBTIs (8) the covariance matrix $\mathbf{C}_{T}$ is computed also using linearization (12). $\mathbf{C}$ is now a $4 N_{T}$-by- $4 N_{T}$ covariance matrix of the elements of vector $\mathbf{R}=$ $[\mathbf{P}, \mathbf{Q}]$, where $\mathbf{Q}=\left[\hat{q}_{0}^{0}, \hat{q}_{0}^{1}, \hat{q}_{1}^{0}, \hat{q}_{1}^{1}, \cdots, \hat{q}_{N_{T}-1}^{0}, \hat{q}_{N_{T}-1}^{1}\right]$. The Jacobian matrix can be expressed by

$$
\mathbf{J}=[\mathbf{K}, \mathbf{L}]=\left[\begin{array}{cccccccc}
\mathbf{K}_{0} & 0 & \cdots & 0 & \mathbf{L}_{0} & 0 & \cdots & 0 \\
0 & \mathbf{K}_{1} & \cdots & 0 & 0 & \mathbf{L}_{1} & \cdots & 0 \\
\vdots & \vdots & \ddots & \vdots & \vdots & \vdots & \ddots & \vdots \\
0 & 0 & \cdots & \mathbf{K}_{N_{T}-1} & 0 & 0 & \cdots & \mathbf{L}_{N_{T}-1}
\end{array}\right]
$$

$\mathbf{K}_{i}$ contains partial derivatives of the invariants $\hat{\mathcal{T}}\left(\mathbf{u}_{i}\right)$ with respect to $\hat{p}_{i}^{0}$ and $\hat{p}_{i}^{1}$ and is given by

$$
\begin{aligned}
\mathbf{K}_{i} \equiv \mathbf{K}_{i, i} & =\left[\frac{\partial \hat{\mathcal{T}}\left(\mathbf{u}_{i}\right)}{\partial \hat{p}_{i}^{0}}, \frac{\partial \hat{\mathcal{T}}\left(\mathbf{u}_{i}\right)}{\partial \hat{p}_{i}^{1}}\right] \\
& =\left[\frac{4 \hat{p}_{i}^{1}}{c_{i}}, \frac{-4 \hat{p}_{i}^{0}}{c_{i}}\right],
\end{aligned}
$$

while $\mathbf{L}_{i}$ contains partial derivatives with respect to $\hat{q}_{i}^{0}$ and $\hat{q}_{i}^{1}$, namely 


$$
\begin{aligned}
\mathbf{L}_{i} \equiv \mathbf{L}_{i, i} & =\left[\frac{\partial \hat{\mathcal{T}}\left(\mathbf{u}_{i}\right)}{\partial \hat{q}_{i}^{0}}, \frac{\partial \hat{\mathcal{T}}\left(\mathbf{u}_{i}\right)}{\partial \hat{q}_{i}^{1}}\right] \\
& =\left[\frac{-2 \hat{q}_{i}^{1}}{e_{i}}, \frac{2 \hat{q}_{i}^{0}}{e_{i}}\right],
\end{aligned}
$$

where $e_{i}=\left[\hat{q}_{i}^{0}\right]^{2}+\left[\hat{q}_{i}^{1}\right]^{2}$.

Equation (12) simplifies to (13) also for PBTIs when discarding redundant coefficients $\hat{\mathbf{q}}_{i}$ from $\mathbf{R}$ that correspond to frequencies $\hat{\mathbf{q}}_{i}=\hat{\mathbf{p}}_{j}$ for some $i, j \in$ $\left\{0,1, \ldots, N_{T}-1\right\}$. The Jacobian matrix (16) has to be organized accordingly: $\mathbf{L}_{i}$ corresponding to redundant coefficients are replaced by $\mathbf{K}_{i, j}$ given by

$$
\begin{aligned}
\mathbf{K}_{i, j} & =\left[\frac{\partial \hat{\mathcal{T}}\left(\mathbf{u}_{i}\right)}{\partial \hat{p}_{j}^{0}}, \frac{\partial \hat{\mathcal{T}}\left(\mathbf{u}_{i}\right)}{\partial \hat{p}_{j}^{1}}\right] \\
& =\left[\frac{-2 \hat{p}_{j}^{1}}{c_{j}}, \frac{2 \hat{p}_{j}^{0}}{c_{j}}\right] .
\end{aligned}
$$

\section{Experiments}

In the experiments, we compared the performance of the weighted and unweighted PBI and PBTI features in classification of blurred and noisy images using nearest neighbour classification. For comparison, we present similar results with and without weighting for the central moment invariants and the phase-tangent invariants [2]. As the phase-tangent invariants are not shift invariant, they are used only in the first experiment. For the moment invariants, we used invariants up to the order 7, as proposed in [2, which results in 18 invariants. For all the frequency domain invariants, we used the invariants for which $\sqrt{u_{1}^{2}+u_{2}^{2}} \leq \sqrt{10}$, but without using the conjugate symmetric or zero frequency invariants. This results also in $N_{T}=18$ invariants.

In the first experiment, the invariants only for blur were considered, namely the PBIs, the phase-tangent invariants, and the central moment invariants (invariant also to shift, but give better results than regular moment invariants [5]).

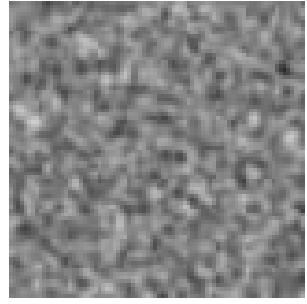

(a)

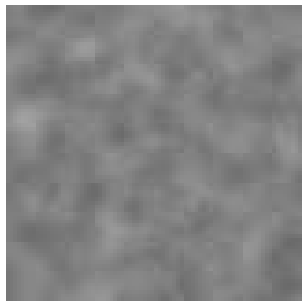

(b)

Fig. 1. (a) An example of the 40 filtered noise images used in the first experiment, and (b) a degraded version of it with blur radius 5 and PSNR $30 \mathrm{~dB}$ 


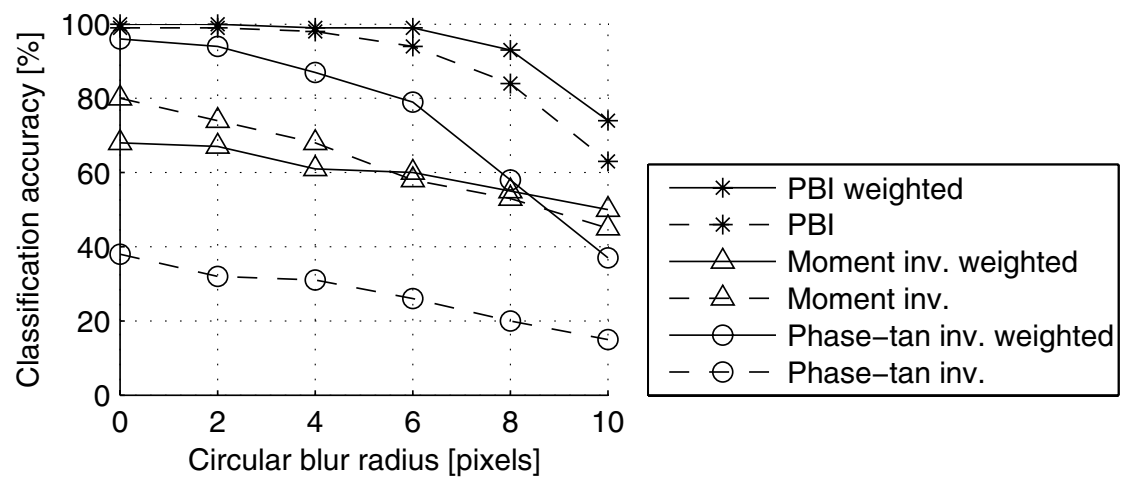

Fig. 2. The classification accuracy of the nearest neighbour classification of the out of focus blurred and noisy (PSNR $20 \mathrm{~dB}$ ) images using various blur invariant features

As test images, we had 40 computer generated images of uniformly distributed noise, which were filtered using a Gaussian low pass filter of size 10-by-10 with the standard deviation $\sigma=1$ to acquire an image, as in Fig. 3.3. that resembles some natural texture. One image at a time was degraded by blur and noise, and was classified as one of the 40 original images using the invariants. The blur was generated by convolving the images with a circular PSF with a radius varying from 0 to 10 pixels with steps of 2 pixels, which models out of focus blur. The PSNR was $20 \mathrm{~dB}$. The image size was cropped finally to 80 -by-80 containing only the valid part of the convolution. The experiment was repeated 20 times for each blur size and for each of the 40 images.

All the tested methods are invariant to circular blur, but there are differences in robustness to noise and boundary error caused by convolution that extends beyond the borders of the observed image. The percentage of correct classification for the three methods, the PBIs, the moment invariants, and the phase-tangent invariants, is shown in Fig. 2 with and without weighting. Clearly, the nonweighted phase-tangent invariants are the most sensitive to disturbances. Their classification result is also improved most by the weighting. The non-weighted moment invariants are known to be more robust to distortions than the corresponding phase-tangent invariants, and this is confirmed by the results. However, the weighting improves the result for moment invariants much less, and only for a blur radii up to 5 pixels making the phase-tangent invariants preferable. Clearly, the best classification results are obtained with the PBIs. Although the PBIs result in the best classification accuracy without weighting, the result is still improved up to $10 \%$ if the weighting is used.

In the second experiment, we tested the blur-translation invariant methods, the PBTIs and the central moment invariants. The test material consisted of $94100 \times 100$ fish images. These original images formed the target classes into which the distorted versions of the images were to be classified. Some 

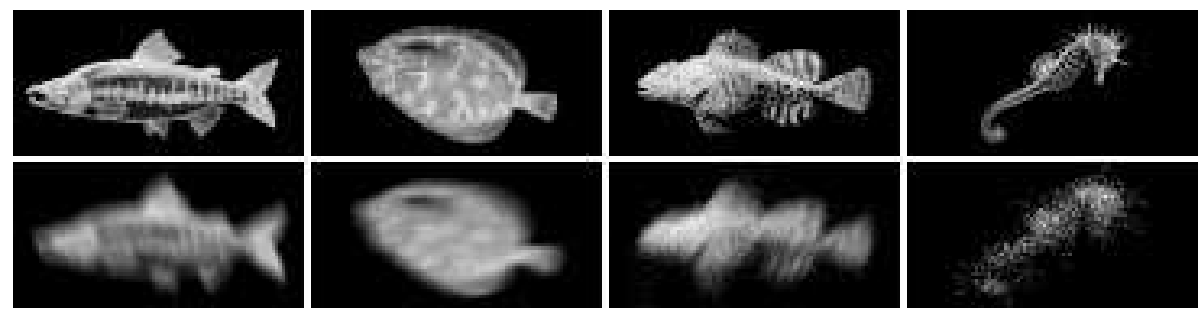

Fig. 3. Top row: four examples of the 94 fish images used in the experiment. Bottom row: motion blurred, noisy, and shifted versions of the same images. The blur length is 6 pixels in a random direction, translation in the range [-5,5] pixels and the PSNRs are from left to right $50,40,30$, and $20 \mathrm{~dB}$. $(45 \times 90$ images are cropped from $100 \times 100$ images.)

original and distorted fish images are shown in Fig. 3. The distortion included linear motion blur of six pixels in a random direction, noise with PSNR from 50 to $10 \mathrm{~dB}$, and random displacement in the horizontal and vertical direction in the range $[-5,5]$ pixels. The objects were segmented from the noisy background before classification using a threshold and connectivity analysis. At the same time, this results in realistic distortion at the boundaries of the objects as some information is lost. The distance between the images of the fish image database was computed using $\mathbf{C}_{T}^{\left(\hat{g}_{1}\right)}$ or $\mathbf{C}_{T}^{\left(\hat{g}_{2}\right)}$ separately instead of their sum $\mathbf{C}_{S}=\mathbf{C}_{T}^{\left(\hat{g}_{1}\right)}+\mathbf{C}_{T}^{\left(\hat{g}_{2}\right)}$, and selecting the larger of the resulting distances, namely distance $=\max \left\{\mathbf{d}^{T}\left[\mathbf{C}_{T}^{\left(\hat{g}_{1}\right)}\right]^{-1} \mathbf{d}, \mathbf{d}^{T}\left[\mathbf{C}_{T}^{\left(\hat{g}_{2}\right)}\right]^{-1} \mathbf{d}\right\}$. This resulted in significantly better classification accuracy for PBTI features (and also for PBI features without displacement of the images), and the result was slightly better also for moment invariants.

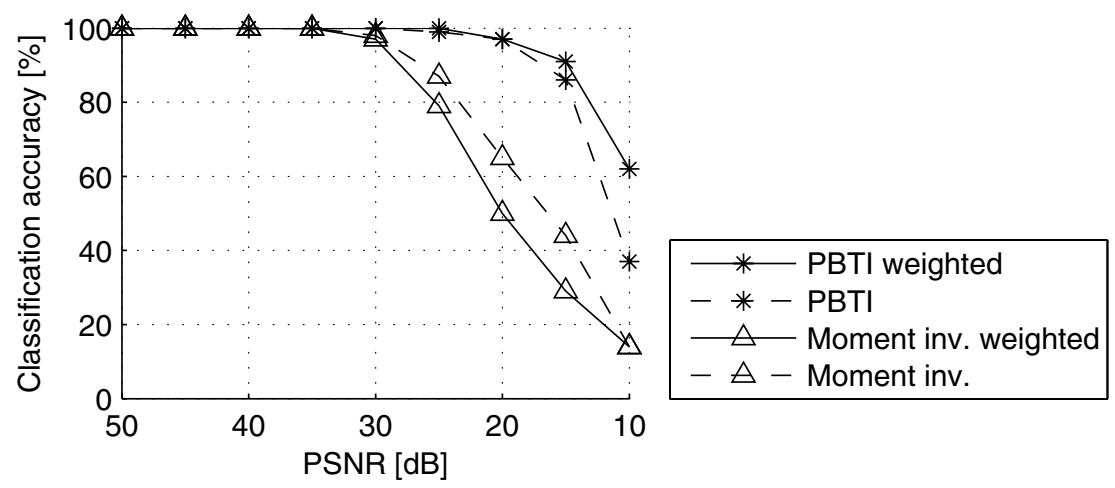

Fig. 4. The classification accuracy of nearest neighbour classification of motion blurred and noisy images using the PBTIs and the moment invariants 
The classification results are shown in the diagram of Fig. 4. Both methods classify images correctly when the noise level is low. When the noise level increases, after $35 \mathrm{~dB}$ the PBTIs perform clearly better than the moment invariants. It can be observed that the weighting does not improve the result of the moment invariants, which is probably due to strong nonlinearity of the moment invariants that cannot be well linearized by (12). However, for the PBTIs the result is improved by up to $20 \%$ through the use of weighting.

\section{Conclusions}

Only few blur invariants have been introduced in the previous literature, and they are based either on image moments or Fourier transform phase. We have shown that the Fourier phase based blur invariants and blur-translation invariants, namely the PBIs and PBTIs, are more robust to noise compared to the moment invariants. In this paper, we introduced a weighting scheme that still improves the results of the Fourier domain blur invariants in classification of blurred images and objects. For the PBIs, the improvement in classification accuracy was up to $10 \%$ and for the PBTIs, the improvement was up to $20 \%$. For comparison, we also showed the results for a similar weighting scheme applied to the moment invariants and the phase-tangent based invariants. The experiments clearly indicated that the weighted PBIs and PBTIs are superior in terms of classification accuracy to other existing methods.

\section{Acknowledgments}

The authors would like to thank the Academy of Finland (project no. 127702), and Prof. Petrou and Dr. Kadyrov for providing us with the fish image database.

\section{References}

1. Wood, J.: Invariant pattern recognition: A review. Pattern Recognition 29(1), 1-17 (1996)

2. Flusser, J., Suk, T.: Degraded image analysis: An invariant approach. IEEE Trans. Pattern Anal. Machine Intell. 20(6), 590-603 (1998)

3. Flusser, J., Zitová, B.: Combined invariants to linear filtering and rotation. Int. J. Pattern Recognition and Artificial Intelligence 13(8), 1123-1136 (1999)

4. Suk, T., Flusser, J.: Combined blur and affine moment invariants and their use in pattern recognition. Pattern Recognition 36(12), 2895-2907 (2003)

5. Ojansivu, V., Heikkilä, J.: Object recognition using frequency domain blur invariant features. In: Ersbøll, B.K., Pedersen, K.S. (eds.) SCIA 2007. LNCS, vol. 4522, pp. 243-252. Springer, Heidelberg (2007)

6. Ojansivu, V., Heikkilä, J.: A method for blur and similarity transform invariant object recognition. In: Proc. International Conference on Image Analysis and Processing (ICIAP 2007), Modena, Italy, September 2007, pp. 583-588 (2007) 
7. Lagendijk, R.L., Biemond, J.: Basic methods for image restoration and identification. In: Bovik, A. (ed.) Handbook of Image and Video Processing, pp. 167-182. Academic Press, London (2005)

8. Ojansivu, V., Heikkilä, J.: Motion blur concealment of digital video using invariant features. In: Blanc-Talon, J., Philips, W., Popescu, D., Scheunders, P. (eds.) ACIVS 2006. LNCS, vol. 4179, pp. 35-45. Springer, Heidelberg (2006) 